\title{
Refractive effects in the scattering of loosely bound nuclei
}

\author{
Florin Carstoiu, ${ }^{1,2,3}$ Livius Trache, ${ }^{1}$ Robert E. Tribble, ${ }^{1}$ and Carl A. Gagliardi ${ }^{1}$ \\ ${ }^{1}$ Cyclotron Institute, Texas A \& M University, College Station, Texas 77843-3366, USA \\ ${ }^{2}$ Laboratoire de Physique Corpusculaire, IN2P3-CNRS, ISMRA et Université de Caen, F-14050 Caen cedex, France \\ ${ }^{3}$ National Institute for Physics and Nuclear Engineering “Horia Hulubei," P.O. Box MG-6, 76900 Bucharest-Magurele, Romania
}

(Received 17 February 2004; published 18 November 2004)

\begin{abstract}
A study of the interaction of the loosely bound nuclei ${ }^{6,7} \mathrm{Li}$ at 9 and $19 \mathrm{MeV} /$ nucleon with light targets has been undertaken. With the determination of unambiguous optical potentials in mind, elastic data for four projectile-target combinations and one neutron transfer reaction ${ }^{13} \mathrm{C}\left({ }^{7} \mathrm{Li},{ }^{8} \mathrm{Li}\right){ }^{12} \mathrm{C}$ have been measured over a large angular range. The kinematical regime encompasses a region where the mean field (optical potential) has a marked variation with mass and energy, but turns out to be sufficiently surface transparent to allow strong refractive effects to be manifested in elastic scattering data at intermediate angles. The identified exotic feature, a "plateau" in the angular distributions at intermediate angles, is fully confirmed in four reaction channels and is interpreted as a prerainbow oscillation resulting from the interference of the barrier and internal barrier far-side scattering subamplitudes.
\end{abstract}

DOI: 10.1103/PhysRevC.70.054610

PACS number(s): 25.70.Bc, 24.10.Ht, 25.70.Hi, 27.20.+n

\section{INTRODUCTION}

The study of nucleus-nucleus elastic scattering has a long history but remains of interest due to both successes and failures that mark it (see, for example, Refs. [1,2] and references therein). It is an important subject per se and is also important as a tool for the description of a series of phenomena that involve the distorted waves given by optical model potentials (OMPs). We are searching here for reliable ways to predict optical model potentials for reactions with radioactive nuclear beams (RNBs). In particular our interest focuses on finding reliable descriptions for transfer reactions involving relatively light, loosely bound nuclei, which are used in indirect methods in nuclear astrophysics. Many RNB studies were made at energies around $10 \mathrm{MeV} /$ nucleon, where the reactions are peripheral, with the intent to obtain information about stellar reaction rates. These reactions use distorted-wave Born approximation (DWBA) techniques to extract nuclear structure information. However, the wellknown existence of many ambiguities in the OMPs extracted from elastic scattering can raise questions about the accuracy of these determinations. Experimental studies using RNBs have, heretofore, not been suitable for detailed elastic scattering analyses. The best information comes from studying the elastic scattering of stable loosely bound nuclei with similar $A$. We chose here to study the elastic scattering of ${ }^{6,7} \mathrm{Li}$ projectiles, because they are fragile (loosely bound), with a pronounced cluster structure and with low $Z$ and can, therefore, exhibit a range of phenomena, involving absorption, diffraction, and refraction, mostly of a nuclear nature.

Earlier we carried out a study of elastic scattering around $10 \mathrm{MeV} /$ nucleon for a range of projectile-target combinations involving $p$-shell nuclei [3]. We found a relatively simple method to predict OMPs for loosely bound nuclei, based on the renormalization of the independent real and imaginary terms obtained from a double-folding procedure using the Jeukenne-Lejeune-Mahaux (JLM) nucleon-nucleon $(N N)$ effective interaction. The procedure successfully de- scribed the data for all projectile-target combinations and energies in the study for most of the angular ranges measured. In two cases $\left({ }^{7} \mathrm{Li}\right.$ at $130 \mathrm{MeV}$ on ${ }^{13} \mathrm{C}$ and ${ }^{6} \mathrm{Li}$ at 99 on ${ }^{12} \mathrm{C}$ ) the folding potentials failed to describe the large-angle data. The results from this work were used to describe elastic scattering angular distributions measured in a series of experiments with RNBs at or around $10 \mathrm{MeV} /$ nucleon: ${ }^{7} \mathrm{Be}$ on ${ }^{10} \mathrm{~B}$ and melamine targets [4], ${ }^{11} \mathrm{C}[5],{ }^{13} \mathrm{~N}[6]$, and ${ }^{17} \mathrm{~F}[7]$ on ${ }^{12} \mathrm{C}$ and ${ }^{14} \mathrm{~N}$ targets. Using a refined analysis, we return to that study here with new data extending the angular ranges for the ${ }^{7} \mathrm{Li}$ scattering and adding data for ${ }^{6} \mathrm{Li}$ scattering.

Recent work [8,9] has established that elastic scattering of light, tightly bound heavy ion systems such as ${ }^{16} \mathrm{O}+{ }^{12} \mathrm{C}$ and ${ }^{16} \mathrm{O}+{ }^{16} \mathrm{O}$ show sufficient transparency for the cross section to be dominated by the far-side scattering. Intermediateangle structures appearing in the elastic scattering distributions at angles beyond the Fraunhofer diffractive region have been identified as Airy minima of a nuclear rainbow-i.e., a destructive interference between two far-side trajectories which sample the interior of the potential. A number of highorder Airy minima have been identified by observing that such structures are largely insensitive to an artificial reduction of the absorption in the optical potential, and therefore they appear as a manifestation of the refractive power of the nuclear potential. While at high energy [10] this picture was well substantiated by a semiclassical nonuniform decomposition of the scattering function [11], at lower energies the situation is more difficult to understand. It has been shown by Anni [12] that such structures could be explained by the interference of two amplitudes appearing in different terms of a multireflection uniform series expansion of the scattering amplitude and therefore the interpretation using rainbow terminology is not appropriate.

For loosely bound nuclei the situation is even more uncertain. When a nucleon or a group of nucleons has small separation energy, the wave function penetrates well beyond the potential range. The corresponding components in the optical potential are expected to be more diffuse as compared 
to normal nuclei, leading to a competition between the increased refractive power of the real potential and increased absorption at the nuclear surface. The small separation energy implies also that the dynamic polarization potential (DPP) [13] arising from the coupling to breakup states may be strong and have a complicated energy and radial dependence. Thus the DPP cannot be treated as a small perturbation for loosely bound nuclei and the usual phenomenological procedure in renormalizing the folding potential form factor may be questioned. It has been estimated that the DPP is strongly repulsive at the nuclear surface in the case of ${ }^{6} \mathrm{Li}$ [14]. This prompted Mahaux, Ngo, and Satchler [15] to conjecture that for loosely bound nuclei the barrier anomaly may be absent due to the cancellation between the repulsive (DPP) and attractive (dispersive) components of the optical potential.

In the specific case of ${ }^{6,7} \mathrm{Li}$ scattering on light targets, a large body of data have been accumulated in the range 5-50 MeV/nucleon. At high energy, Nadasen and his group $[16,17]$ have been able to derive a unique optical potential which was essential to assess the quality of the folding model. At lower energies, ambiguities found in the analysis of data prevented any definite conclusion about the strength and energy dependence of the optical potential. A study by Trcka et al. [18] on ${ }^{6} \mathrm{Li}+{ }^{12} \mathrm{C}$ elastic scattering at $50 \mathrm{MeV}$ found an exotic feature ("plateau") in the angular distribution of the elastic scattering at intermediate angles which resembles similar structures found in more bound systems. They interpreted the structure as a diffractive effect arising from an angular-momentum-dependent absorption. There are experimental hints that such structures also appear in neighboring systems ${ }^{6} \mathrm{Li}+{ }^{16} \mathrm{O}$ and ${ }^{6} \mathrm{Li}+{ }^{9} \mathrm{Be}$ as a possible manifestation of the average properties of the interaction potential.

In this paper we present an analysis of elastic scattering of ${ }^{6,7} \mathrm{Li}$ on ${ }^{12,13} \mathrm{C}$ and ${ }^{9} \mathrm{Be}$ targets at 9 and $19 \mathrm{MeV} /$ nucleon. The lower energy was chosen in view of our systematic studies of nuclear reactions for astrophysics. The higher energy is close to the saturation energy for these projectiles-i.e., the energy where almost all reaction channels are open. The "plateau" feature is confirmed in four projectile-target combinations at $9 \mathrm{MeV} /$ nucleon. The high selectivity induced by this structure allowed the derivation of an almost unique Woods-Saxon optical potential. A folding model analysis using the complex, density- and energy-dependent $N N$ interaction of JLM [19], where corrections due to the strong DPP have been included, confirmed that our elastic distributions could be described using deep and extremely transparent potentials. The remaining ambiguities have been eliminated using an accurate dispersion relation analysis. The intermediate-angle structures have been discussed using the semiclassical uniform approximation for the scattering function of Brink and Takigawa [20]. We explain the intermediate-angle structure as a coherent interference effect of two subamplitudes corresponding to trajectories reflected at the barrier and interfering with trajectories which sample the nuclear interior. Thus, this refractive effect appears as a signature of a highly transparent interaction potential.

The paper is structured in the following way: after this introduction, the experimental methods are discussed in Sec. II, the analysis of the elastic scattering data using phenom-
TABLE I. List of the elastic scattering experiments presented in this paper.

\begin{tabular}{cccc}
\hline \hline No. & Reaction & $E[\mathrm{MeV}]$ & $\theta_{\text {lab }}[\mathrm{deg}]$ \\
\hline 1 & ${ }^{6} \mathrm{Li}+{ }^{12} \mathrm{C}$ & 54 & $2-56$ \\
2 & ${ }^{6} \mathrm{Li}+{ }^{13} \mathrm{C}$ & 54 & $2-59$ \\
3 & ${ }^{7} \mathrm{Li}+{ }^{9} \mathrm{Be}$ & 63 & $4-52$ \\
4 & ${ }^{7} \mathrm{Li}+{ }^{13} \mathrm{C}$ & 63 & $4-56$ \\
5 & ${ }^{7} \mathrm{Li}+{ }^{9} \mathrm{Be}$ & 130 & $4-47$ \\
6 & ${ }^{7} \mathrm{Li}+{ }^{13} \mathrm{C}$ & 130 & $4-47$ \\
\hline \hline
\end{tabular}

enological and microscopic optical model potentials is discussed in Sec. III, and the implications of this analysis for the transfer reaction $\left({ }^{7} \mathrm{Li},{ }^{8} \mathrm{Li}\right)$ are discussed in Sec. IV. In Sec. V the dispersion relation is used to put additional constraints on the potentials extracted, followed by a discussion of the decomposition of the far-side scattering amplitude into barrier and internal barrier components responsible for the "plateau" structure at intermediate angles (Sec. VI) and the conclusions (Sec. VII).

\section{EXPERIMENTS}

The experiments were performed using ${ }^{6} \mathrm{Li}$ and ${ }^{7} \mathrm{Li}$ beams of 9 and $19 \mathrm{MeV} /$ nucleon from the Texas A\&M University K500 superconducting cyclotron and the Multipole Dipole Multipole (MDM) magnetic spectrometer [21]. A list of the measurements is given in Table I. The measurements with ${ }^{7} \mathrm{Li}$ were done to extend the angular range covered in earlier work. The experimental setup and the data reduction procedures were similar to those used in Ref. [3]. The beams were prepared using the beam analysis system [22], which allows for the control of the energy spread $(\Delta E / E$ up to $1 / 2500)$ and angular spread $\left(0.1^{\circ}\right)$ of the beam. Self-supporting ${ }^{9} \mathrm{Be}\left(200 \mu \mathrm{g} / \mathrm{cm}^{2}\right.$ thick $),{ }^{12} \mathrm{C}\left(260 \mu \mathrm{g} / \mathrm{cm}^{2}\right)$, and ${ }^{13} \mathrm{C}$ $\left(390 \mu \mathrm{g} / \mathrm{cm}^{2}\right)$ targets were placed perpendicular to the beam in the target chamber of the MDM. The magnetic field of the MDM spectrometer was set to transport fully stripped Li ions to the focal plane where they were observed in the modified Oxford detector [23]. In the detector, the position of the particles along the dispersive direction was measured with resistive wires at four different depths, separated by about $16 \mathrm{~cm}$ each. For particle identification we used the specific energy loss measured in the ionization chamber and the residual energy measured in a NE102A plastic scintillator located behind the output window of the detector. The input and output windows of the detector were made of 1.8- and $7.2 \mathrm{mg} / \mathrm{cm}^{2}$-thick Kapton foils, respectively. The ionization chamber was filled with pure isobutane at 40 torr. The entire horizontal acceptance of the spectrometer, $\Delta \theta= \pm 2^{\circ}$, and a restricted vertical opening, $\Delta \phi= \pm 0.5^{\circ}$, were used in the measurements at forward angles, whereas at the largest angles the vertical opening of the acceptance window was raised to $\Delta \phi= \pm 1.0^{\circ}$. Ray tracing was used to reconstruct the scattering angle. For this purpose, position calibration of the detector was performed using scattering from a thin $\mathrm{Au}$ target $\left(212 \mu \mathrm{g} / \mathrm{cm}^{2}\right)$ and an angle mask consisting of five open- 


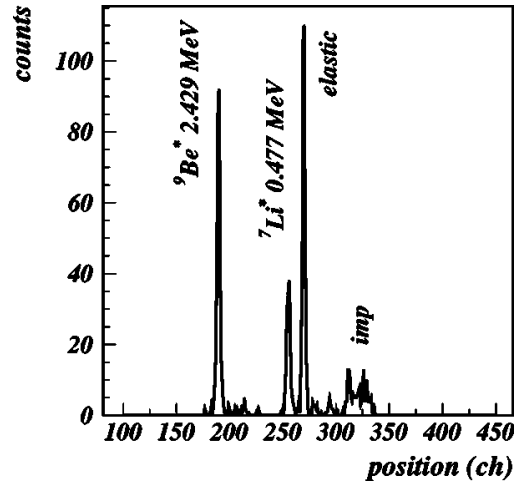

FIG. 1. Spectrum from the scattering of ${ }^{7} \mathrm{Li}$ on the ${ }^{9} \mathrm{Be}$ target at $63 \mathrm{MeV}$ and $\theta_{l a b}=15.25^{\circ} \pm 0.25^{\circ}$. The angle was chosen at a minimum in the angular distribution of the elastic cross section to emphasize inelastic excitations. The peaks labeled "imp" are from scattering on a small amount of heavier impurities in the target.

ings of $\delta \theta=0.1^{\circ}$, located at $-1.6^{\circ},-0.8^{\circ}, 0^{\circ},+0.8^{\circ}$, and $+1.6^{\circ}$ relative to the central angle of the spectrometer. In addition to RAYTRACE [24] calculations, angle calibration data were obtained at several angles by using the angle mask. Typically the spectrometer was moved by $2^{\circ}$ or $3^{\circ}$ at a time to allow for an angle overlap that provided a self-consistency check of the data. Normalization of the data was done using current integration in a Faraday cup. Focal plane reconstruction was done at each angle using the position measured with the signals in the wire nearest to the focal plane and using the detector angle obtained from the position measured at two of the four wires (typically the first and last). The angular range $\Delta \theta=4^{\circ}$ covered by the acceptance slit was divided into eight equal bins.

The measurements with the angle mask showed that the resolution in the scattering angle (laboratory) was $\Delta \theta_{\text {res }}$ $=0.18^{\circ}-0.25^{\circ}$ full width at half maximum (FWHM), which includes a contribution from the angular spread of the beam of about $0.1^{\circ}$. The best energy resolution obtained at forward angles was 120-150 keV FWHM. It degraded at larger angles due to the kinematic factor $k=(1 / p) d p / d \theta$ coupled with the finite angular spread in the beam. However, the resolution was always sufficient to separate elastic and inelastic scattering. An example of the spectra measured is shown in Fig. 1. The active length of the focal plane allowed us to cover a total excitation energy of about $7 \mathrm{MeV}$, centered around the elastic peak. Thus we were able to measure inelastic scattering to the lowest excited states of the projectile-target systems at the same time. In one of the experiments we also measured the neutron transfer reaction ${ }^{13} \mathrm{C}\left({ }^{7} \mathrm{Li},{ }^{8} \mathrm{Li}\right){ }^{12} \mathrm{C}$ at $E\left({ }^{7} \mathrm{Li}\right)=63 \mathrm{MeV}$, which was discussed elsewhere in detail [25] and is used here to check the sensitivity of observables in other channels to the OMP extracted from the elastic scattering data.

To obtain accurate absolute values for the cross sections, target thickness and charge collection factors were determined by a two-target method as described in Ref. [26]. We also determined the target thickness by measuring the energy loss of alpha particles from a ${ }^{228} \mathrm{Th}$ source. Combining the results of these independent determinations, we conclude that we have an overall normalization accuracy of $7 \%$ for the absolute values of the cross sections.
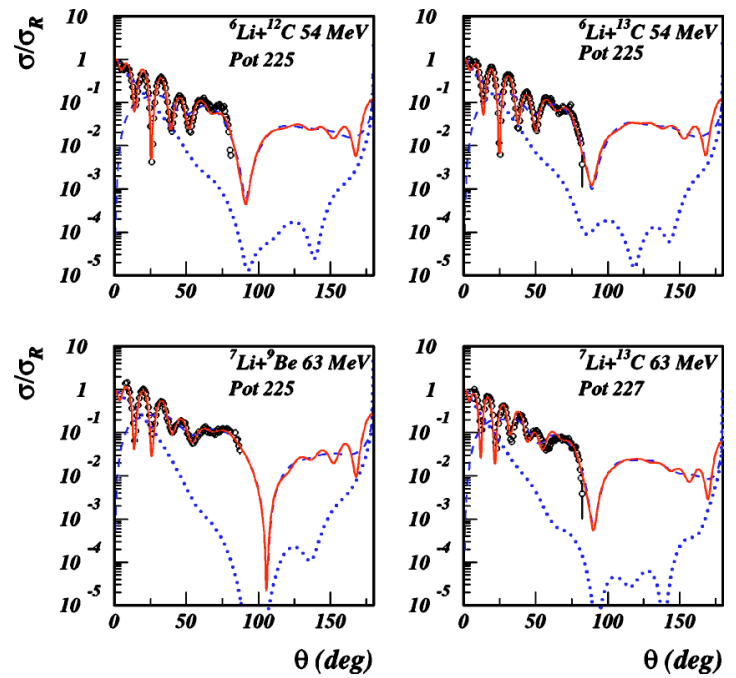

FIG. 2. (Color online) Woods-Saxon optical model analysis (solid lines) of elastic scattering data (open points) at $9 \mathrm{MeV} /$ nucleon (Table II). Far-side and near-side cross sections are also shown by dashed and dotted lines, respectively. The depth of the real potential is shown to identify the particular WS potential parameters used in the calculations.

\section{OPTICAL MODEL ANALYSIS}

The measured elastic scattering data at $9 \mathrm{MeV} /$ nucleon, shown in Fig. 2 as the ratio to the Rutherford cross section, extend to a larger angular range than previously reported [3]. These data show complex forms with characteristic rapid oscillations at small angles followed by a marked change in shape at intermediate angles: a plateau develops at $\theta$ $=50^{\circ}-70^{\circ}$ which is followed by a deep minimum at $\theta$ $\approx 80^{\circ}$. Assuming pure Fraunhofer scattering at forward angles, we extract a grazing angular momentum $l_{g} \approx 15$ from the angular spacing $\Delta \theta=\pi /\left(l_{g}+1 / 2\right)$. The striking fact is that the same pattern emerges for all four projectile-target combinations, including that for the ${ }^{9} \mathrm{Be}$ target where a much stronger absorption is expected.

Similarities seen in the differential cross sections shown in Fig. 2 indicate general wave-mechanical characteristics of the scattering process and average systematic properties of the nuclear interaction. Specific structure effects can be isolated only as small deviations from the normal behavior. Therefore the data are analyzed using optical potentials with conventional Woods-Saxon (WS) form factors for the nuclear term, supplemented with a Coulomb potential generated by a uniform charge distribution with a reduced radius fixed to $r_{c}=1 \mathrm{fm}$. No preference has been found for volumeor surface-localized absorption and throughout the paper only volume absorption is considered. In the absence of any spin-dependent observables, spin-orbit or tensor interactions have been ignored. Ground-state reorientation couplings also have been neglected. The potential is defined by six parameters specifying the depth and geometry of the real and imaginary terms, using standard notation, as given in Ref. [3]. The number of data points per angular distribution exceeds $N=100$ and therefore the usual goodness-of-fit criterion $\left(\chi^{2}\right)$ normalized to $N$ has been used. A source of bias 
TABLE II. Best fit Woods-Saxon parameters. Reduced radii are defined in the heavy ion convention. All lengths are given in fm, depths and energies in $\mathrm{MeV}$, cross sections in $\mathrm{mb}$, and volume integrals in $\mathrm{MeV} \mathrm{fm}{ }^{3}$. The Coulomb-reduced radius is fixed to $r_{c}=1 \mathrm{fm} . R_{V}$ and $R_{W}$ are the rms radii of the real and imaginary potentials, respectively.

\begin{tabular}{|c|c|c|c|c|c|c|c|c|c|c|c|c|c|}
\hline Reaction & $\begin{array}{l}\text { Energy } \\
{[\mathrm{MeV}]}\end{array}$ & $\begin{array}{c}V_{0} \\
{[\mathrm{MeV}]}\end{array}$ & $\begin{array}{c}W_{0} \\
{[\mathrm{MeV}]}\end{array}$ & $\begin{array}{c}r_{V} \\
{[\mathrm{fm}]}\end{array}$ & $\begin{array}{c}r_{W} \\
{[\mathrm{fm}]}\end{array}$ & $\begin{array}{c}a_{V} \\
{[\mathrm{fm}]}\end{array}$ & $\begin{array}{c}a_{W} \\
{[\mathrm{fm}]}\end{array}$ & $\chi^{2}$ & $\begin{array}{c}\sigma_{R} \\
{[\mathrm{mb}]}\end{array}$ & $\begin{array}{c}J_{V} \\
{\left[\mathrm{MeV} \mathrm{fm}^{3}\right]}\end{array}$ & $\begin{array}{c}R_{V} \\
{[\mathrm{fm}]}\end{array}$ & $\begin{array}{c}J_{W} \\
{\left[\mathrm{MeV} \mathrm{fm}^{3}\right]}\end{array}$ & $\begin{array}{c}R_{W} \\
{[\mathrm{fm}]}\end{array}$ \\
\hline \multirow[t]{2}{*}{${ }^{6} \mathrm{Li}+{ }^{12} \mathrm{C}$} & 54 & 225.47 & 15.75 & 0.503 & 1.157 & 0.900 & 0.737 & 17.71 & 1309 & 338 & 3.70 & 121 & 4.59 \\
\hline & & 371.31 & 17.70 & 0.439 & 1.109 & 0.856 & 0.777 & 13.60 & 1322 & 419 & 3.47 & 125 & 4.56 \\
\hline \multirow[t]{2}{*}{${ }^{6} \mathrm{Li}+{ }^{13} \mathrm{C}$} & 54 & 225.28 & 14.75 & 0.502 & 1.181 & 0.916 & 0.707 & 14.62 & 1327 & 327 & 3.76 & 114 & 4.63 \\
\hline & & 364.46 & 16.95 & 0.443 & 1.133 & 0.871 & 0.744 & 14.24 & 1338 & 403 & 3.53 & 119 & 4.58 \\
\hline \multirow[t]{2}{*}{${ }^{7} \mathrm{Li}+{ }^{9} \mathrm{Be}$} & 63 & 225.85 & 24.74 & 0.536 & 0.941 & 0.828 & 0.980 & 10.14 & 1456 & 369 & 3.49 & 146 & 4.66 \\
\hline & & 368.34 & 29.38 & 0.478 & 0.882 & 0.790 & 1.004 & 11.85 & 1470 & 464 & 3.28 & 153 & 4.62 \\
\hline \multirow[t]{2}{*}{${ }^{7} \mathrm{Li}+{ }^{13} \mathrm{C}$} & 63 & 227.94 & 15.37 & 0.529 & 1.186 & 0.932 & 0.669 & 20.09 & 1367 & 328 & 3.87 & 107 & 4.64 \\
\hline & & 278.86 & 24.19 & 0.594 & 1.050 & 0.789 & 0.721 & 20.04 & 1334 & 411 & 3.53 & 126 & 4.38 \\
\hline${ }^{7} \mathrm{Li}+{ }^{13} \mathrm{C}$ & 130 & 149.11 & 29.73 & 0.636 & 0.932 & 0.885 & 0.929 & $2.61^{\mathrm{a}}$ & 1403 & 282 & 3.90 & 132 & 4.62 \\
\hline${ }^{7} \mathrm{Li}+{ }^{9} \mathrm{Be}$ & 130 & 143.41 & 33.64 & 0.581 & 0.829 & 0.892 & 1.094 & $3.03^{\mathrm{a}}$ & 1446 & 295 & 3.76 & 169 & 4.80 \\
\hline${ }^{13} \mathrm{C}+{ }^{9} \mathrm{Be}$ & 130 & 159.85 & 24.43 & 0.674 & 0.983 & 0.868 & 0.914 & 13.69 & 1552 & 280 & 3.96 & 104 & 4.79 \\
\hline
\end{tabular}

${ }^{\mathrm{a}}$ Uniform $10 \%$ errors.

was the finite angular acceptance of the detectors (the $0.5^{\circ}$ bins, in the present case). The averaging associated with this finite angular resolution has most effect on the depth of sharp minima. A few exploratory calculations showed that allowing the normalization to vary did not result in any qualitative changes and did not indicate that any renormalization by more than a few percent would be preferred. Optical parameter sets collected from the literature were used as starting values for the search procedure. In particular the potential OM1 of Trcka et al. [18] has been extensively tested. Guided by these potentials and by our earlier analysis [3] a number of some $10^{6}$ potentials with real volume integrals in the range $J_{V}=200-600 \mathrm{MeV} \mathrm{fm}^{6}$ have been generated for each reaction channel, thus exploring the functional Woods-Saxon space in full detail. Local minima were identified and a complete search on all six parameters determined the best fit potentials. The plateau feature at intermediate angles and the sharp decrease in the cross section near $\theta=80^{\circ}$ could be fit only with deep potentials with real volume integrals (per pairs of interacting nucleon) exceeding a critical value $J_{V \text { crit }} \approx 300 \mathrm{MeV} \mathrm{fm}^{2}$. There is a consistent preference for potentials with relatively weak imaginary parts, with values of $W$ around $15 \mathrm{MeV}$, except for ${ }^{7} \mathrm{Li}$ scattering where somewhat larger values are needed to fit the data. We systematically find $r_{V}<r_{W}$ and large diffuseness parameters $a_{V} \simeq a_{w}$ $\simeq 0.8 \mathrm{fm}$ in agreement with theoretical expectations for loosely bound nuclei $[27,28]$. A grid search procedure of the real depth of the potential allowed us to identify discrete ambiguities. The parameters for the first two discrete families are given in Table II. These are identified by a jump of $\Delta J_{V} \approx 100 \mathrm{MeV} \mathrm{fm}^{3}$ from one family to the next and an almost constant imaginary volume integral. As a consequence, the total reaction cross section seems to be a well-determined observable. Gridding on other WS parameters revealed a continuous ambiguity of the form $J_{V} R_{V} \approx$ const, where $R_{V}$ is the rms radius of the potential. The larger the volume integral, the smaller the radius that is required to fit the data. This is a clear manifestation of a complicated radial depen- dence of the DPP which may lead to radii much smaller than the minimal value implied by the folding model (e.g., $R_{F}^{2}$ $=R_{1}^{2}+R_{2}^{2}$, for a zero-range $N N$ effective interaction). However, for each discrete family rather precise values of the rms radii were required to fit both forward- and intermediateangle cross sections.

Sometimes more subjective criteria may be used to choose between various ambiguous potentials based upon general theoretical expectations. For example, one may require consistency with the results of analyses of other data for the same system at nearby energies with the expectation that the potential should not change rapidly with mass and energy. Individual elastic data sets possess individual idiosyncrasies which facilitate the inference of a single local potential. We note that, seemingly, there is a compatibility between all data sets: an optimum potential found for one data set gives already a good fit to the other. In fact, potentials given as first entry in Table II were obtained by iterating several times the procedure described above in an attempt to find a single potential which would simultaneously fit all data at $9 \mathrm{MeV} /$ nucleon. A compromise could be obtained with transparent deep potentials close to $V_{0} \approx 225 \mathrm{MeV}$ having a strongly refractive core at small radii, surrounded by a weakly absorptive halo. In fact, examining the ratio $w(r)$ $=W(r) / V(r)$ [29] as a function of the radial distance, we found that our potential has internal $(r \sim 0-4 \mathrm{fm})$ and surface $(r>8 \mathrm{fm})$ transparency $(w \approx 0.1)$ but with a pronounced maximum $(w \approx 0.8)$ near the empirical strong absorption radius $\left(R_{s} \approx 6 \mathrm{fm}\right)$, in agreement with the systematics found in other more bound systems [29]. The surface-localized absorption suggests that the reaction mechanism is dominated by direct reactions. The relatively large radius of the absorption required by the data is an indication that fusion already sets in the region of the barrier and that fusion is a large component of the total reaction cross section. Unfortunately, little information exists on the total reaction cross sections for ${ }^{6,7} \mathrm{Li}$ projectiles on $\mathrm{C}$ and $\mathrm{Be}$ targets. In the one case where a comparison is possible, the cross section predicted 
TABLE III. Best fit JLM1 parameters. The notations are those from the text. Lengths are given in fm, energies in MeV, cross sections in $\mathrm{mb}$, and volume integrals in $\mathrm{MeV} \mathrm{fm}^{3}$.

\begin{tabular}{|c|c|c|c|c|c|c|c|c|c|c|c|}
\hline Reaction & $\begin{array}{l}\text { Energy } \\
{[\mathrm{MeV}]}\end{array}$ & $\begin{array}{c}t_{V} \\
{[\mathrm{fm}]}\end{array}$ & $\begin{array}{c}t_{W} \\
{[\mathrm{fm}]}\end{array}$ & $N_{V}$ & $N_{W}$ & $\chi^{2}$ & $\begin{array}{c}\sigma_{R} \\
{[\mathrm{mb}]}\end{array}$ & $\begin{array}{c}J_{V} \\
{\left[\mathrm{MeV} \mathrm{fm}^{3}\right]}\end{array}$ & $\begin{array}{c}R_{V} \\
{[\mathrm{fm}]}\end{array}$ & $\begin{array}{c}J_{W} \\
{\left[\mathrm{MeV} \mathrm{fm}^{3}\right]}\end{array}$ & $\begin{array}{c}R_{W} \\
{[\mathrm{fm}]}\end{array}$ \\
\hline \multirow[t]{10}{*}{${ }^{6} \mathrm{Li}+{ }^{12} \mathrm{C}$} & 30 [39] & 0.30 & 2.45 & 0.60 & 0.46 & 14.8 & 1371 & 396 & 3.66 & 72 & 4.93 \\
\hline & 50 [11] & 0.08 & 2.78 & 0.56 & 0.78 & 12.0 & 1315 & 373 & 3.64 & 120 & 4.42 \\
\hline & 54 & 0.08 & 2.76 & 0.54 & 0.77 & 21.4 & 1556 & 351 & 3.64 & 116 & 5.11 \\
\hline & 90 [40] & 0.70 & 2.70 & 0.52 & 1.24 & 18.4 & 1591 & 313 & 3.73 & 173 & 4.96 \\
\hline & 99 [41] & 0.60 & 1.75 & 0.47 & 1.01 & 4.21 & 1225 & 277 & 3.69 & 145 & 4.27 \\
\hline & 124 [42] & 0.60 & 1.75 & 0.51 & 1.09 & 3.96 & 1243 & 292 & 3.69 & 168 & 4.28 \\
\hline & 156 [43] & 0.50 & 1.50 & 0.50 & 0.94 & 7.98 & 1146 & 271 & 3.66 & 154 & 4.19 \\
\hline & 168 [42] & 0.60 & 1.75 & 0.58 & 1.11 & 5.87 & 1231 & 305 & 3.68 & 185 & 4.28 \\
\hline & 210 [16] & 0.20 & 1.35 & 0.56 & 0.93 & 23.5 & 1062 & 276 & 3.59 & 161 & 4.05 \\
\hline & 318 [17] & 0.80 & 1.95 & 0.60 & 0.85 & 9.00 & 1069 & 251 & 3.69 & 148 & 4.35 \\
\hline${ }^{6} \mathrm{Li}+{ }^{13} \mathrm{C}$ & 54 & 0.08 & 2.76 & 0.54 & 0.77 & 21.4 & 1556 & 351 & 3.64 & 116 & 5.11 \\
\hline${ }^{6} \mathrm{Li}+{ }^{16} \mathrm{O}$ & 50 [18] & 0.50 & 2.81 & 0.55 & 0.60 & 13.4 & 1643 & 346 & 3.64 & 91 & 5.24 \\
\hline${ }^{7} \mathrm{Li}+{ }^{9} \mathrm{Be}$ & 63 & 0.09 & 1.20 & 0.46 & 0.98 & 19.5 & 1538 & 274 & 3.64 & 152 & 4.80 \\
\hline${ }^{7} \mathrm{Li}+{ }^{13} \mathrm{C}$ & 63 & 0.12 & 2.59 & 0.52 & 0.78 & 19.0 & 1652 & 335 & 3.74 & 113 & 5.07 \\
\hline${ }^{7} \mathrm{Li}+{ }^{13} \mathrm{C}$ & $130^{\mathrm{a}}$ & 0.13 & 1.97 & 0.48 & 1.02 & 4.58 & 1392 & 280 & 3.73 & 146 & 4.50 \\
\hline${ }^{7} \mathrm{Li}+{ }^{9} \mathrm{Be}$ & $130^{\mathrm{a}}$ & 0.12 & 2.34 & 0.50 & 1.23 & 7.98 & 1404 & 304 & 3.62 & 183 & 4.65 \\
\hline${ }^{14} \mathrm{~N}+{ }^{13} \mathrm{C}$ & 162 & 1.44 & 1.82 & 0.39 & 0.73 & 33.1 & 1563 & 220 & 4.29 & 89 & 4.66 \\
\hline \multirow[t]{2}{*}{${ }^{10} \mathrm{~B}+{ }^{9} \mathrm{Be}$} & 100 & 1.89 & 1.02 & 0.30 & 1.01 & 6.9 & 1266 & 185 & 4.33 & 146 & 4.08 \\
\hline & & 0.47 & 2.28 & 0.48 & 0.93 & 29.6 & 1558 & 298 & 3.75 & 133 & 4.79 \\
\hline
\end{tabular}

${ }^{\mathrm{a}}$ Uniform $10 \%$ errors.

by the optical potential in Table III for ${ }^{6} \mathrm{Li}$ at $35 \mathrm{MeV} /$ nucleon is fully consistent with the experimental values obtained by Fukuda et al. [30] for ${ }^{6} \mathrm{Li}$ at $38 \mathrm{MeV} /$ nucleon on $\mathrm{C}$ and $\mathrm{Be}$ targets. The reaction cross sections listed in Tables II and III also agree very well at all energies with the results of calculations for the total reaction cross sections using simplified strong absorption [31] or Glauber models [32].

A variety of notch tests have been performed to determine the radial sensitivity of the potential. One test was done using a Gaussian spike superimposed on the real potential at a given radius. It shows that there is a relatively high sensitivity for radial distances as low as 4-6 fm, well inside the strong absorption radius. Deeper inside this radial range, the refractive index, defined as $n=\sqrt{1-V / E_{\text {c.m. }}}$, is almost real and reaches values as high as $n=2.6$, comparable to that of diamond.

As mentioned already, it was shown in Refs. [8-10] that the elastic scattering of light heavy ion systems such as ${ }^{16} \mathrm{O}+{ }^{12} \mathrm{C}$ and ${ }^{16} \mathrm{O}+{ }^{16} \mathrm{O}$ shows sufficient transparency for the cross section to be dominated by far-side scattering. Structures appearing in the elastic scattering angular distributions at intermediate angles have been identified as Airy minima of a nuclear rainbow, due to a destructive interference between two far-side trajectories which sample the interior of the potential. At $19 \mathrm{MeV} /$ nucleon the ${ }^{7} \mathrm{Li}$ scattering data show rapid, diffractive Fraunhofer oscillations at small angles due to the strong near-far amplitude interference (Fig. 3). Beyond the crossover the near-side amplitude makes a negligible contribution to the cross section. The shoulder and deep minimum seen at $9 \mathrm{MeV} /$ nucleon (Fig. 2) are washed out in the far-side amplitude and only a broad, less pronounced minimum survives, followed by a broad Airy maximum and an exponential, structureless decay of the cross section at large angles. Clearly, both the data at 9 and $19 \mathrm{MeV} /$ nucleon (Figs. 2 and 3) show far-side dominance as a possible manifestation of refractive effects. However, this simple dominance does not explain, by itself, the difference in the angu-

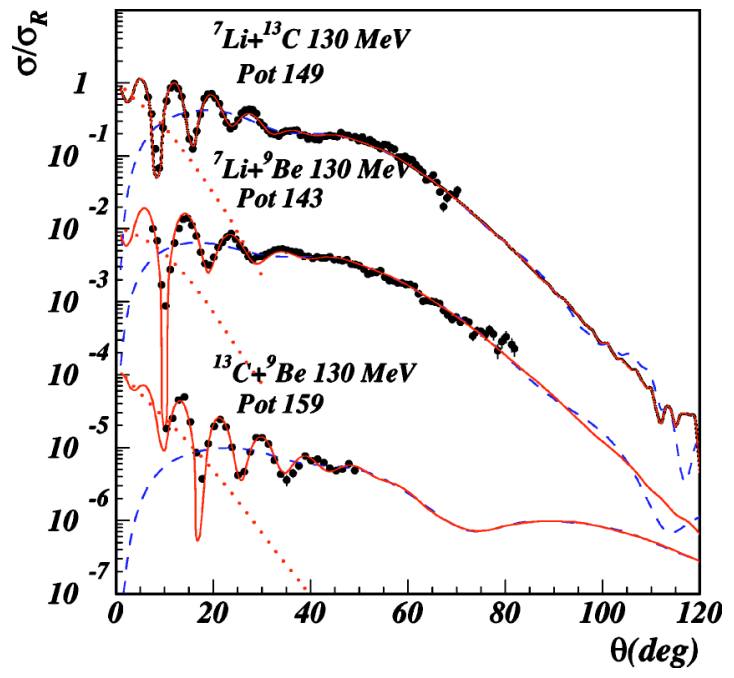

FIG. 3. (Color online) Woods-Saxon optical model analysis of elastic data at $130 \mathrm{MeV}$ (Table II). Far-side and near-side cross sections are also shown by dashed and dotted lines, respectively. The lower curves are multiplied by $10^{-2}$ and $10^{-4}$, respectively. 
lar distributions seen at these energies, suggesting a difference in the reaction mechanism. In fact the above picture has already been challenged by Anni [12] and by Michel et al. [33] for the simple reason that the far-side amplitude has never been decomposed in subamplitudes which would explain the interference. We come back to this topic in Sec. VI. For the moment we adopt the interpretation of Michel et al. [33] and denote the complex structure at intermediate angles in our data as prerainbow oscillations.

In the remainder of this section we discuss the ability of the folding model to describe the prerainbow oscillation seen at $9 \mathrm{MeV} /$ nucleon and the rainbow patterns at higher energies. Data at somewhat lower energies are also examined in order to see if the plateau feature persists in adjacent systems and on a larger energy range. Our preferred model is the nuclear matter approach of JLM [19] which incorporates a complex, energy- and density-dependent parametrization of the $N N$ effective interaction obtained in a Brueckner HartreeFock approximation from the Reid soft-core nucleonnucleon potential. The systematic study [3] of the elastic scattering between $p$-shell nuclei at energies around $10 \mathrm{MeV} /$ nucleon led to the surprising result that, on average, the imaginary part of the folded JLM potential was perfectly adequate to describe such reactions and did not need any renormalization $\left(N_{W}=1.00 \pm 0.09\right)$, while the real component needed a strong renormalization, in line with other effective interactions used in folding models. However, the present data extend to a much larger angular range and need further refinements of this model.

In the JLM model the complex form factor for the optical potential is given by

$$
U(R)=\int d \vec{r}_{1} d \vec{r}_{2} \rho_{1}\left(r_{1}\right) \rho_{2}\left(r_{2}\right) v(\rho, E, s) g(s),
$$

where $v$ is the (complex) $N N$ interaction, $\rho_{1(2)}$ are the singleparticle densities of the interacting partners, calculated in a standard spherical Hartree-Fock procedure using the energy density functional of Beiner and Lombard with the surface term adjusted to reproduce the total binding energy [34,35], $\vec{s}=\vec{r}_{1}+\vec{R}-\vec{r}_{2}$ is the $N N$ separation distance between interacting nucleons, and $\rho$ is the overlap density. The smearing function $g(s)$ is taken as a normalized Gaussian [3,19,36],

$$
g(s)=\frac{1}{t^{3} \pi^{3 / 2}} \exp \left(-s^{2} / t^{2}\right),
$$

which tends to a $\delta$ function for $t \rightarrow 0$, while for finite values of the range parameter $t$ it increases the rms radius of the folding form factor by $r_{g}^{2}=(3 / 2) t^{2}$, leaving unchanged the volume integral. Inclusion of a smearing function with a varying range parameter greatly increases the ability of the folding form factor to simulate the radial dependence of the DPP.

The geometric or arithmetic mean of the overlapping densities has been used to define the overlap density $\rho$ in Eq. (1):

$$
\rho=\left[\rho_{1}\left(\vec{r}_{1}+\frac{1}{2} \vec{s}\right) \rho_{2}\left(\vec{r}_{2}-\frac{1}{2} \vec{s}\right)\right]^{1 / 2}
$$

and

$$
\rho=\frac{1}{2}\left[\rho_{1}\left(\vec{r}_{1}+\frac{1}{2} \vec{s}\right)+\rho_{2}\left(\vec{r}_{2}-\frac{1}{2} \vec{s}\right)\right] .
$$

The former was introduced by Campi and Sprung in densitydependent Hartree-Fock calculations [37]. It is physically appealing since the overlap density tends to zero when one of the interacting nucleons is far from the bulk and to the nuclear matter saturation value at complete overlap. The approximation in Eq. (4) is similar to that used in folding calculations with density-dependent M3Y effective interactions [38], except for the factor of $1 / 2$ which has been introduced here because the JLM interaction is defined only up to the nuclear matter saturation value $\rho \leqslant \rho_{0}$. The optical model analysis presented above showed clearly that the prerainbow oscillations (at $9 \mathrm{MeV} /$ nucleon) and rainbow patterns (at $9 \mathrm{MeV} /$ nucleon) could be described if and only if the potentials have the proper rms radius. It turns out that the smearing procedure described above is essential in simulating the complicated radial dependence of the dynamic polarization potential.

In the earlier analysis [3], fixed values for the range parameters $t_{V}=1.2 \mathrm{fm}$ and $t_{W}=1.75 \mathrm{fm}$, found from a global analysis of the data, were used. Only the renormalization factors $N_{V}$ and $N_{W}$ were left free in the fits for each case. In the present analysis with double-folded potentials, all four parameters - two strength parameters $\left(N_{V}\right.$ and $\left.N_{W}\right)$ and two range parameters $\left(t_{V}\right.$ and $\left.t_{W}\right)$ - have been searched simultaneously to fit the data for each case,

$$
U_{\mathrm{DF}}(r)=N_{V} V\left(r, t_{V}\right)+i N_{W} W\left(r, t_{W}\right),
$$

to obtain a phenomenological representation of the DPP as a uniform renormalization of the depths and radii of the folding potentials. The calculations using Eqs. (3) and (4) are dubbed JLM1 and JLM2, respectively. As these give very similar results only JLM1 parameters are listed in Table III and the results of the calculations are shown in Figs. 4-7. At $9 \mathrm{MeV} /$ nucleon (Fig. 4) the same pattern emerges as with Woods-Saxon form factors. The prerainbow oscillation is carried entirely by the dominant far-side component. Some other high-order structures appear at angles near $180^{\circ}$ as the result of near- and far-amplitude interference. At most forward angles this interference produces an inner Fraunhofer crossing which gives rise to a deep minimum in the cross section.

For ${ }^{7} \mathrm{Li}+{ }^{9} \mathrm{Be}$ at $63 \mathrm{MeV}$, the JLM1 calculation failed to describe the oscillation near $\theta=80^{\circ}$ for the simple reason that the data required a rms radius for the real potential of $R_{V}$ $=3.4 \mathrm{fm}$, while the bare JLM interaction predicts a minimal $R_{V}=3.6 \mathrm{fm}$ for $t_{V} \approx 0$. This once again reflects the critical role played by the radial behavior of DPP. This is also illustrated in the upper right quadrant of Fig. 5 where two JLM solutions for the reaction ${ }^{10} \mathrm{~B}+{ }^{9} \mathrm{Be}$ at $10 \mathrm{MeV} /$ nucleon are indicated (see also Table III). The solution with a smaller real volume integral which better fits the forward angles predicts a smooth, exponentially decaying cross section beyond $\theta$ 

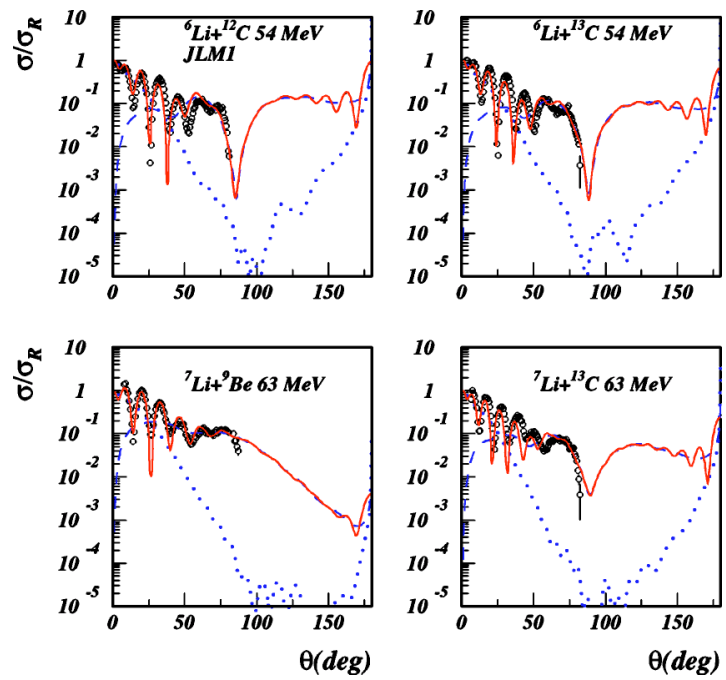

FIG. 4. (Color online) Comparison of the JLM1 folding model calculations (solid lines) with present data at $9 \mathrm{MeV} /$ nucleon. The parameters are given in Table III. Far-side (dashed) and near-side (dotted) cross sections are indicated in the form of ratios to the Rutherford cross sections.

$\approx 60^{\circ}$. The second solution with a real volume integral close to the critical value $J_{V \text { crit }} \approx 300 \mathrm{MeV} \mathrm{fm}{ }^{3}$ gives rise to a shallow prerainbow oscillation at these angles (not covered by experiments). The high selectivity of the prerainbow oscillations to the optical potentials is also illustrated in Fig. 6 where other lower-energy ${ }^{6} \mathrm{Li}$ scattering data from the literature are explored. The ${ }^{6} \mathrm{Li}+{ }^{12} \mathrm{C}$ data at $50 \mathrm{MeV}$ [18] could be described in the whole angular range only with potentials exceeding the critical value of the real volume integral found before. In Fig. 7 we show ${ }^{6} \mathrm{Li}+{ }^{12} \mathrm{C}$ elastic scattering data at
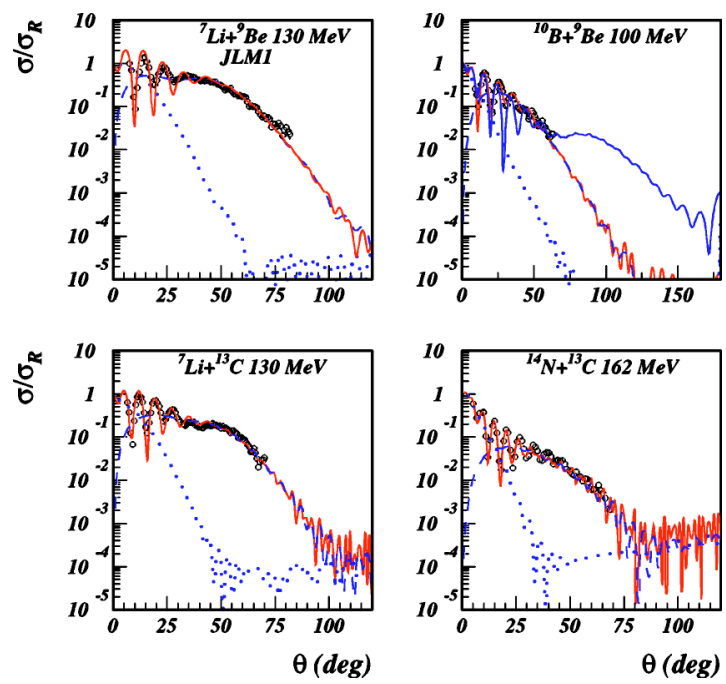

FIG. 5. (Color online) Comparison of JLM1 folding model calculations with ${ }^{7} \mathrm{Li}$ scattering data at $19 \mathrm{MeV} /$ nucleon (left panels). On the right, scattering of ${ }^{10} \mathrm{~B}$ and ${ }^{14} \mathrm{~N}$ (data from Ref. [3]) are shown. Two JLM1 solutions are indicated for the ${ }^{10} \mathrm{~B}+{ }^{9} \mathrm{Be}$ reaction. The parameters are given in Table III. Far-side (dashed) and nearside (dotted) cross sections are indicated in ratio to Rutherford cross sections.
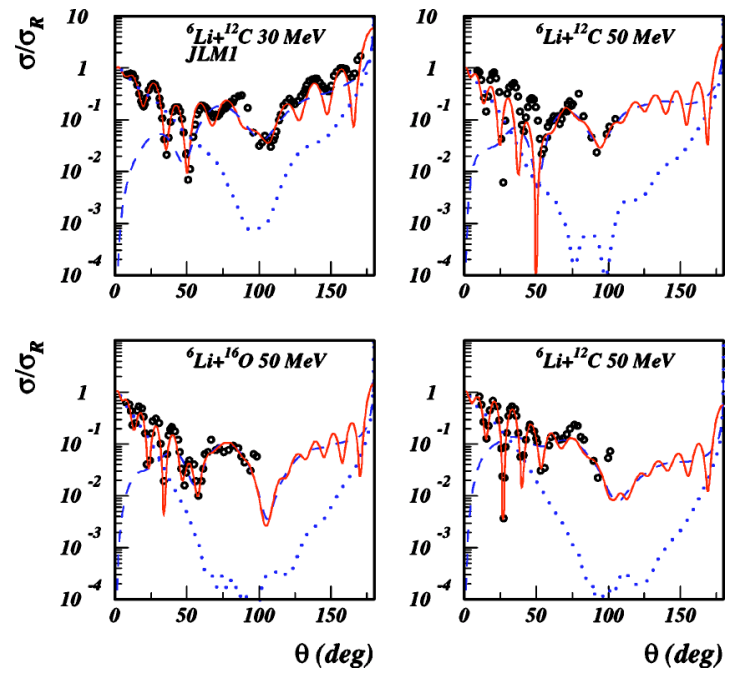

FIG. 6. (Color online) Comparison of the JLM1 folding model calculations with ${ }^{6} \mathrm{Li}$ scattering data on light targets at 30 and $50 \mathrm{MeV}$ laboratory energy (data from Refs. [18,39]). For the ${ }^{12} \mathrm{C}$ target at $50 \mathrm{MeV}$, only a solution with a real volume integral exceeding the critical value $J_{V}=300 \mathrm{MeV} \mathrm{fm}^{3}$ (Table III) is able to reproduce both forward and intermediate angles (right bottom panel). Far-side (dashed lines) and near-side (dotted) cross sections are indicated in ratio to Rutherford cross sections.

seven energies between 15 and $50 \mathrm{MeV} /$ nucleon. Now, even at high energy (Fig. 7) the JLM1 description of the rainbow patterns is exemplary [to be compared with Figs. 6(a) and 6(c) of Ref. [3]]. This suggests that the geometrical details of the optical potential rather than the density dependence are essential for a correct description of ${ }^{6,7} \mathrm{Li}$ elastic scattering at low and intermediate energies.

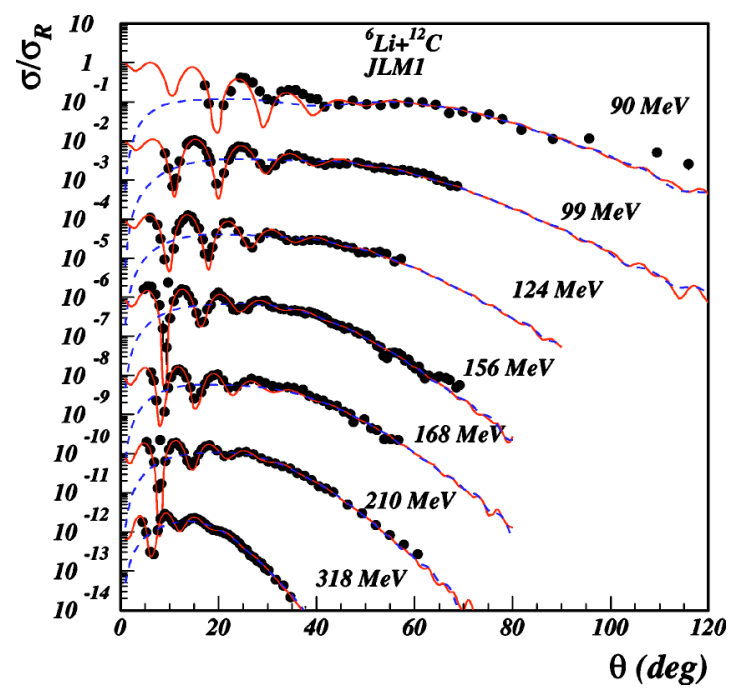

FIG. 7. (Color online) Comparison of JLM1 folding model calculations (solid lines) with high-energy ${ }^{6} \mathrm{Li}$ scattering data. The sources of data and calculation parameters are given in Table III. The far-side (dashed lines) cross sections are indicated in ratio to the Rutherford cross sections (and from the top curve below, each case is multiplied by an extra $10^{-2}$ factor). The near-side cross section (not shown) is important only at the most forward angles. 
A close examination of the parameters in Table III reveals an erratic variation of the range of parameters $t_{V(W)}$ from one energy to another and from system to system. As mentioned above, this largely reflects the mass and energy dependence of the DPP. The other parameters are more stable. The strength parameter $N_{V}$ decreases slowly from 5 to $16.5 \mathrm{MeV} /$ nucleon and then increases again up to $53 \mathrm{MeV} /$ nucleon, the highest energy at which reliable data exist. This may suggest that the DPP reaches its maximum amplitude at energies around $16 \mathrm{MeV} /$ nucleon. On average the $N_{V}$ values in Table III are somewhat larger than in our earlier analysis [3], reflecting the need for stronger refractive effects, but again $N_{W}$ approaches unity, on average.

\section{TRANSFER REACTION}

As already mentioned, one measurement included the neutron transfer reaction ${ }^{13} \mathrm{C}\left({ }^{7} \mathrm{Li},{ }^{8} \mathrm{Li}\right){ }^{12} \mathrm{C}$ at $E\left({ }^{7} \mathrm{Li}\right)$ $=63 \mathrm{MeV}$. The purpose of the study was to determine the asymptotic normalization coefficients (ANC) for the ground state of ${ }^{8} \mathrm{Li}$ and, then, using charge symmetry, to relate it to that in its mirror nucleus ${ }^{8} \mathrm{~B}$ in order to calculate the astrophysical factor $S_{17}$ that gives the rate of the proton capture reaction $\left.{ }^{7} \mathrm{Be}(p, \gamma)\right)^{8} \mathrm{~B}$, of crucial importance for the solar neutrino problem. The major advantage of the neutron transfer reaction over its mirror proton transfer reaction is that it involves a stable beam and, therefore, a much more precise and detailed angular distribution could be measured. That allowed the determination of the admixture of the minor $1 p_{1 / 2}$ component in the wave function of the ground state of ${ }^{8} \mathrm{Li}$ (and ${ }^{8} \mathrm{~B}$, respectively) which is dominated by the $1 p_{3 / 2}$ orbital. The results of this experiment were reported in Ref. [25]. In that study we paid particular attention to the dependence of the results on the optical model potentials used in the entrance and exit channels.

Eleven different combinations of entrance and exit potentials were used to show that the resulting values for $C_{p_{3 / 2}}^{2}$ and $C_{p_{1 / 2}}^{2}$ are very stable, when the potentials are reasonable. The potentials used were either volume Woods-Saxon forms with the parameters from similar projectile-target combinations at similar energies or were obtained from the double-folding procedure with the renormalization coefficients from the previous paper [3]. Calculations done after the publication with the new (deeper) potential " 227 " of Table III in both entrance and exit channels lead to minor $(\sim 5 \%)$ variations in the results. The very good agreement between the experimental data and the DWBA calculations and between the results of present and previous calculations (Fig. 8) shows that the region of the potential contributing to transfer (the surface) is well described. This simultaneous description of elastic and transfer data is also an argument for the complete determination of the optical potentials.

\section{DISPERSION RELATION}

The dispersion relation is a fundamental property of the optical potential (see, for example, [44]) and a selection between ambiguous potentials can be performed by studying the dispersive properties of these potentials, provided accu-

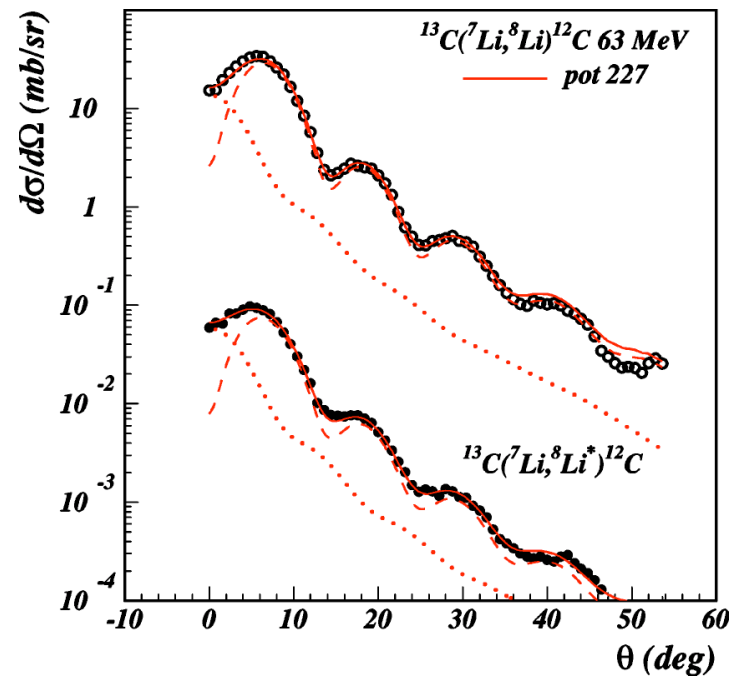

FIG. 8. (Color online) The angular distributions for the neutron transfer reaction ${ }^{13} \mathrm{C}\left({ }^{7} \mathrm{Li},{ }^{8} \mathrm{Li}\right){ }^{12} \mathrm{C}$ to the ground (top, open points) and first excited state (bottom, solid points) of ${ }^{8} \mathrm{Li}$. The calculations shown (solid line) are done using the potential "227" in Table II. The data are shown as points and the separate contributions of the $p_{1 / 2} \rightarrow p_{3 / 2}$ (dashed line) and $p_{1 / 2} \rightarrow p_{1 / 2}$ (dotted line) components are shown in both cases.

rate analyses of experimental data are available over a large energy range.

The threshold anomaly which manifests itself as a sharp increase of the real optical potential for energies close to the Coulomb barrier has been explained by Nagarajan, Mahaux, and Satchler [44] as due to the opening of reaction channels with increasing energy. An application of the dispersion relation for elastic scattering of ${ }^{16} \mathrm{O}$ on ${ }^{208} \mathrm{~Pb}$ at energies around $80 \mathrm{MeV}$ accounted well for this effect. Later it was conjectured by Mahaux, Ngo, and Satchler [15] that for loosely bound nuclei, this anomaly may be absent. Recent studies of the threshold anomaly in ${ }^{6,7} \mathrm{Li}$-induced reactions lead to contradictory conclusions: a cancellation between the attractive (dispersive) component and the repulsive dynamic polarization potential $[45,46]$, dynamic polarization potentials of opposite sign for ${ }^{6,7} \mathrm{Li}$ [47], and breakup suppression above the barrier energies [48].

Therefore, the energy dependence of the ${ }^{6,7} \mathrm{Li}$ optical potential is far from clear and the competition between dispersive (attractive) and coupling to continuum (repulsive) effects need to be studied more carefully. An earlier study [49] showed that the total reaction cross section for ${ }^{6} \mathrm{Li}$ scattering saturates at energies around $20 \mathrm{MeV} /$ nucleon and therefore dispersive effects could be identified by accumulating good optical potentials in this energy range. The real and imaginary volume integrals for the optical potentials obtained in the previous sections are plotted in Fig. 9. Both WoodsSaxon and JLM folding results have been included. These are supplemented with values derived from the smooth OM1 potential of Trcka et al. [18].

We assume that the local optical potential may be written as $V=V_{0}+\Delta V(E)$ where $V_{0}$ is independent of energy and $\Delta V(E)$ is the energy-dependent DPP. We ignore the spurious energy dependence of $V_{0}$ arising from nonlocality which is 


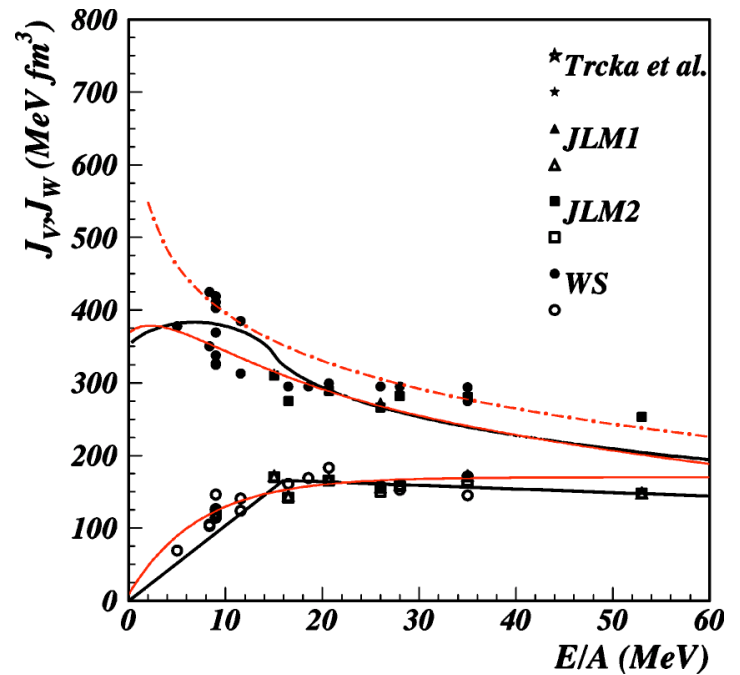

FIG. 9. (Color online) Energy dependence of the real (solid points) and imaginary (open points) volume integrals obtained in the analyses with Woods-Saxon and folding (JLM) optical potentials. The stars show the values obtained from the OM1 optical potential of Ref. [18]. The curves for $J_{V}$ are the result from the dispersion relation, normalized to the empirical value at $26 \mathrm{MeV} /$ nucleon, assuming the schematic models shown for $J_{W}$. The dash-dotted line gives the empirical energy dependence of the real volume integral of Ref. [50].

expected to be weak for heavy ions. We use the dispersion relation connecting the imaginary and real volume integrals in the subtracted form

$$
J_{\Delta V, E_{s}}(E)=\left(E-E_{s}\right) \frac{\mathcal{P}}{\pi} \int \frac{J_{W}\left(E^{\prime}\right)}{\left(E^{\prime}-E_{s}\right)\left(E^{\prime}-E\right)} d E^{\prime},
$$

where $E_{s}$ is a reference energy and $\mathcal{P}$ is the principal value of the integral. In principle the evaluation of this equation requires knowledge of $J_{W}$ values at all energies. The above subtracted form takes advantage of the fact that the energy dependence of $J_{W}$ far from saturation energy is not very important and the unknown contributions are absorbed by normalizing to the empirical value at a convenient reference energy:

$$
J_{\Delta V, E_{s}}(E)=J_{\Delta V}(E)-J_{\Delta V}\left(E_{s}\right) .
$$

Two schematic models have been employed here to estimate the energy dependence of the imaginary volume integral. A first one approximates this energy dependence by straight line segments [15], which makes the evaluation of Eq. (6) analytical. A more realistic energy dependence is given by

$$
J_{W}(E)=J_{W}^{0}[1-\beta \exp (-\alpha E)],
$$

where the parameters $J_{W}^{0}=170 \mathrm{MeV} \mathrm{fm}^{3}, \alpha=0.023 \mathrm{MeV}^{-1}$, and $\beta=0.95$ describe better the energy dependence in the important range $0-20 \mathrm{MeV} /$ nucleon. In both calculations the reference energy was set to $E_{s}=156 \mathrm{MeV}$, an energy where the JLM folding model gives precise values for volume integrals. In general, the calculated dispersion contributions get more repulsive as the energy increases and the cor- responding real potentials get shallower, in qualitative consistency with phenomenology. An empirical logarithmic dependence of the form $J_{V}=-785+95 \ln (E)$ has been found in Ref. [50] mostly based on unique OM potentials determined from 35 and $53 \mathrm{MeV} /$ nucleon ${ }^{6} \mathrm{Li}$ scattering on light targets. This matches perfectly the dependence obtained with the dispersion relation for $E>10 \mathrm{MeV} /$ nucleon, but disagrees at lower energies. In fact, this logarithmic dependence is physically meaningful and can be understood on the basis of the dispersion relation with a schematic (line segments) approach for the imaginary volume integral.

A relatively strong localized energy variation is predicted by the linear model in the range $0-20 \mathrm{MeV} /$ nucleon, while the exponential model predicts a smooth dependence on the entire range of energies. This last calculation is much closer to the data and seems to confirm $J_{V}=320 \mathrm{MeV} \mathrm{fm}^{3}$ as the most realistic value at $9 \mathrm{MeV} /$ nucleon, in surprising agreement with values found for the more bound system ${ }^{16} \mathrm{O}$ $+{ }^{16} \mathrm{O}$ (see, e.g., Fig. 6 in Ref. [51]). Most likely the phenomenological values found at $5 \mathrm{MeV} /$ nucleon are due to the erratic variation in the WS parameters due to the rapidly changing elastic scattering angular distributions [52] near the resonance energy region around $20 \mathrm{MeV}$.

\section{SEMICLASSICAL BARRIER AND INTERNAL BARRIER AMPLITUDES}

Once we have established the main features of the average OM potential, we turn now to study the reaction mechanism in the elastic scattering of ${ }^{6,7} \mathrm{Li}$ on light targets at $9 \mathrm{MeV} /$ nucleon using semiclassical methods. The far-side dominance observed in the angular distributions at 9 and $19 \mathrm{MeV} /$ nucleon is not able to explain the differences in the reaction mechanism at these energies. The reason is of course that the far/near $(\mathrm{F} / \mathrm{N})$ decomposition method does not perform a dynamic decomposition of the scattering function, but merely decomposes the scattering amplitude into traveling waves. The intermediate-angle structures, such as those observed in our angular distributions, have been repeatedly interpreted as arising from the interference of two ranges in angular momenta, $\ell_{<}$and $\ell_{>}$, contributing to the same negative deflection angle. However, the corresponding cross sections $\sigma_{F<}$ and $\sigma_{F>}$ cannot be isolated because their dynamic content ( $S$ matrix) is not accessible.

The semiclassical uniform approximation for the scattering amplitude of Brink and Takigawa [20] is well adapted to describe situations in which the scattering is controlled by at most three active, isolated, complex turning points. An approximate multireflection series expansion of the scattering function can be obtained, the terms of which have the same simple physical meaning as in the exact Debye expansion for the scattering of light on a spherical well. The major interest in this theory comes from the fact that it can give precious information on the response of a nuclear system to the nuclear interior. Recent application [12] of this technique helped to clarify the controversial problem of the "Airy oscillation" seen in low-energy ${ }^{16} \mathrm{O}+{ }^{12} \mathrm{C}$ scattering [8].

For the potentials in Table II (the first entry for each of the four cases measured here) we discard the absorptive terms and define the effective potential as 

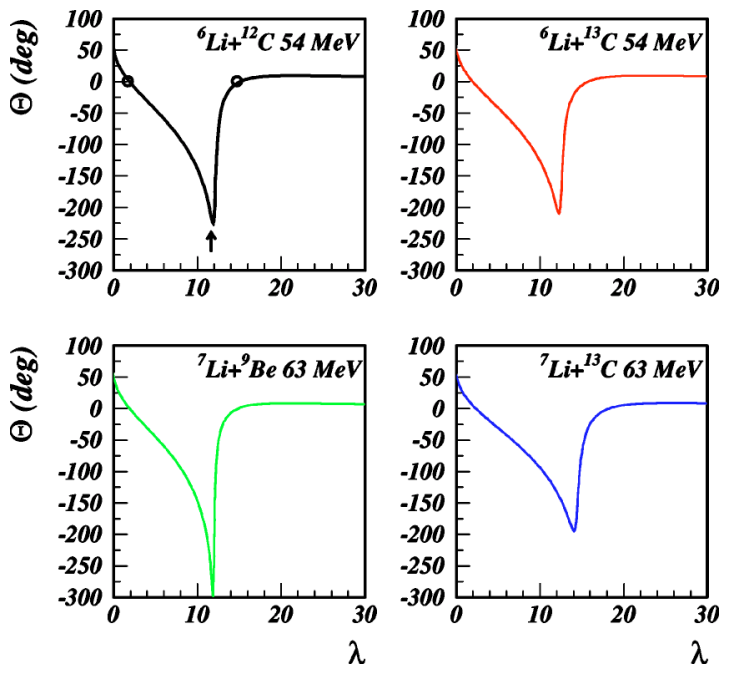

FIG. 10. (Color online) Semiclassical deflection functions for the real potentials shown in Table II. In the top left quad, glories are indicated by open dots and the orbiting angular momentum by an arrow.

$$
V_{e f f}(r)=V(r)+\frac{\hbar^{2}}{2 \mu} \frac{\lambda^{2}}{r^{2}}, \quad \lambda=\ell+\frac{1}{2},
$$

where the Langer prescription has been used for the centrifugal term. This guarantees the correct behavior of the semiclassical wave function at the origin [53]. Then we calculate the deflection function

$$
\Theta(\lambda)=\pi-2 \int_{r_{1}}^{\infty} \frac{\sqrt{\frac{\hbar^{2}}{2 \mu}} \lambda d r}{r^{2} \sqrt{E_{\mathrm{c} . \mathrm{m} .}-V_{e f f}}},
$$

where $r_{1}$ is the outer zero of the square root-i.e., the radius of closest approach to the scatterer-and $\mu$ is the reduced mass. Note that with the replacement $\hbar \lambda=b \sqrt{2 \mu E}$, Eq. (10) becomes identical to the classical deflection function $\Theta(b)$, where $b$ is the impact parameter. The results are shown in Fig. 10. The behavior of $\Theta(\lambda)$ is the one expected for a strong nuclear potential in a near-orbiting kinematical situation in which the c.m. energy approximately equals that of the top of the barrier for some specific angular momentum. The deflection functions exhibit no genuine minima, but rather a pronounced cusp close to an orbiting logarithmic singularity. Therefore any interpretation of structures in angular distributions in terms of Airy oscillations can be discarded. Rather we need an interpretation appropriate for orbiting, a well-documented situation in classical physics [54]. We identify the cusp angular momenta as orbiting momenta $\left(\lambda_{o}\right)$ since they are related to the coalescence of two (barrier) turning points and the innermost turning point given by the centrifugal barrier becomes classically accessible. There are two branches that can be distinguished: an internal branch for low active momenta $\lambda<\lambda_{o}$ related to semiclassical trajectories which penetrate into the nuclear pocket and a less developed external (barrier) branch $\left(\lambda>\lambda_{o}\right)$ related to trajectories deflected at the diffuse edge of the potential.

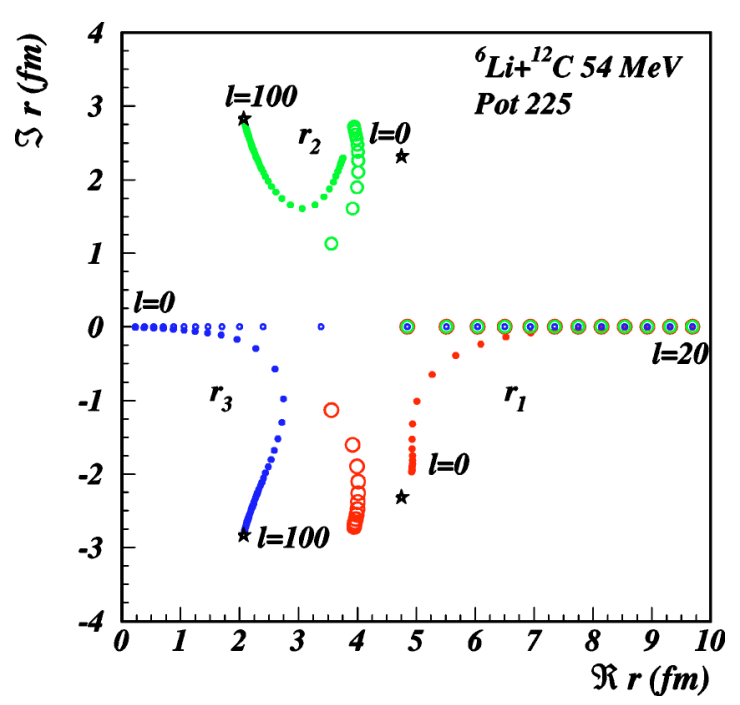

FIG. 11. (Color online) Complex turning points (solid symbols) for the potential " 225 " shown in Table II at integer angular momenta. Open symbols denote turning points for the real potential alone. Stars indicate complex poles of the potential.

However, this simple calculation cannot determine the relative importance of these branches and provides no information about the interference effects of the corresponding semiclassical trajectories. To clarify these points it is best to go into the complex $r$ plane and look for complex turning points-i.e., the complex roots of the quantity $E_{\text {c.m. }}-V_{\text {eff }}$ $-i W$. This is an intricate numerical problem, because, for a WS optical potential, the turning points are located near the potential singularities and there are an infinite number of such poles. The situation for integer angular momenta is depicted in Fig. 11 for the reaction ${ }^{6} \mathrm{Li}+{ }^{12} \mathrm{C}$ at $54 \mathrm{MeV}$ using the potential " 225 " in Table II. Only turning points nearest the real axis are retained and we observe an ideal situation with three, well-isolated, turning points for each partial wave. Even small absorption plays an essential role in the motion of turning points. Removing the imaginary part $W$, the barrier turning points $\left(r_{1,2}\right)$ become complex conjugates while the internal turning point is purely real (open symbols in Fig. 11).

The multireflection expansion of the scattering function in the Brink-Takigawa approach reads

$$
S_{W K B}(\ell)=\sum_{q=0}^{\infty} S_{q}(\ell)
$$

where

$$
S_{0}(\ell)=\frac{\exp \left(2 i \delta_{1}^{\ell}\right)}{N\left(S_{21} / \pi\right)}
$$

and, for $q \neq 0$, 


$$
S_{q}(\ell)=(-)^{q+1} \frac{\exp \left[2 i\left(q S_{32}+S_{21}+\delta_{1}^{\ell}\right)\right]}{N^{q+1}\left(S_{21} / \pi\right)} .
$$

In these equations $\delta_{1}^{\ell}$ is the WKB (complex) phase shift corresponding to the turning point $r_{1}, N(z)$ is the barrier penetrability factor,

$$
N(z)=\frac{\sqrt{2 \pi}}{\Gamma\left(z+\frac{1}{2}\right)} \exp (z \ln z-z),
$$

and $S_{i j}$ is the action integral calculated between turning points $r_{i}$ and $r_{j}$ :

$$
S_{i j}=\int_{r_{i}}^{r_{j}} d r\left\{\frac{2 \mu}{\hbar^{2}}\left[E_{\mathrm{c} . \mathrm{m} .}-V_{e f f}-i W\right]\right\}^{1 / 2} .
$$

$S_{21}$ and $S_{32}$ are independent of the integration path provided they lie on the first Riemann sheet and collision with potential poles is avoided. Each term in Eq. (11) has a simple physical interpretation. The first term (the barrier term, denoted also $S_{B}$ ) retains contributions from trajectories reflected at the barrier, not penetrating the internal region. The $q$ th term corresponds to trajectories refracted $q$ times in the nuclear interior with $q-1$ reflections at the barrier turning point $r_{2}$. Summation of terms $q \geqslant 1$ can be recast into a single term,

$$
S_{I}=\frac{\exp \left[2 i\left(S_{32}+S_{21}+\delta_{1}^{\ell}\right)\right]}{N\left(S_{21} / \pi\right)^{2}} \frac{1}{1+\exp \left[2 i S_{32}\right] / N\left(S_{21} / \pi\right)},
$$

and is known as the internal barrier scattering function. When the absorption in the nuclear interior is large, the second factor in the above equation reduces to 1 and we are left with the expression used in [33]. Since the semiclassical scattering function is decomposed additively, $S_{W K B}=S_{B}+S_{I}$, the corresponding total scattering amplitude is decomposed likewise as $f_{W K B}=f_{B}+f_{I}$ and conveniently the corresponding barrier and internal barrier angular distributions are calculated as $\sigma_{B, I}=\left|f_{B, I}\right|^{2}$, using the usual angular momentum expansion of the amplitudes.

The accuracy of the semiclassical calculation has been checked by comparing the barrier and internal barrier absorption profiles with the exact quantum-mechanical result in Fig. 12. First, one observes that the semiclassical B/I expansion is an exact decomposition of the quantum result. They are virtually identical at the scale of the figure. The internal component gets significant values up to the grazing angular momentum $\left(\ell_{g}=15\right)$ and is negligibly small beyond this value. The barrier component resembles a strong absorption profile and this justifies the interpretation that it corresponds to that part of the flux not penetrating into the nuclear interior. For values near the orbiting angular momentum $\left(\ell_{o}\right.$ $\approx 12$ ), the two components interfere and a downward spike appears in the total profile, in complete agreement with the quantum result. Second, the B/I components are almost decoupled in the angular momentum space and therefore they will contribute in different angular ranges.

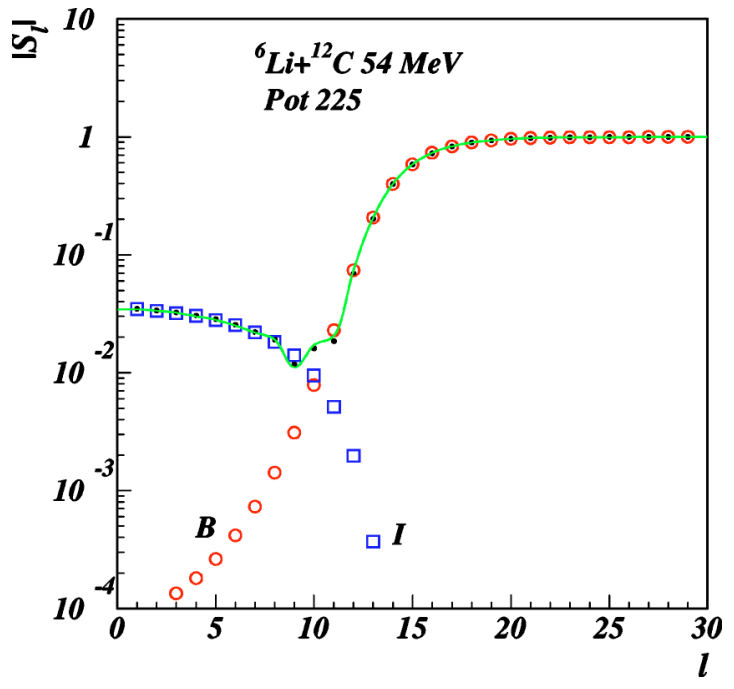

FIG. 12. (Color online) Semiclassical decomposition of the scattering function for the WS potential of Fig. 11. Barrier (open circles) and internal barrier components (squares) are indicated. The exact total quantum $S$ matrix is indicated by small dots. The line is a cubic spline interpolation of the total semiclassical scattering function for the same potential.

Semiclassical cross sections are compared with the data in Fig. 13 for the reaction ${ }^{6} \mathrm{Li}+{ }^{12} \mathrm{C}$ at $54 \mathrm{MeV}$. Better insight into this technique is obtained by further decomposing the $\mathrm{B} / \mathrm{I}$ components into far and near $(\mathrm{BF} / \mathrm{BN}$ and $\mathrm{IF} / \mathrm{IN})$ subcomponents. Clearly, the barrier component dominates the forward-angle region. Fraunhofer diffractive oscillations appear as the result of $\mathrm{BF}$ and $\mathrm{BN}$ interference. At large angles, the internal contribution accounts for the full cross section. As both B/I contributions are dominated by the far-side component (Fig. 13, bottom panels), we show in Fig. 14 the angles at which the phase difference of the BF and IF am-
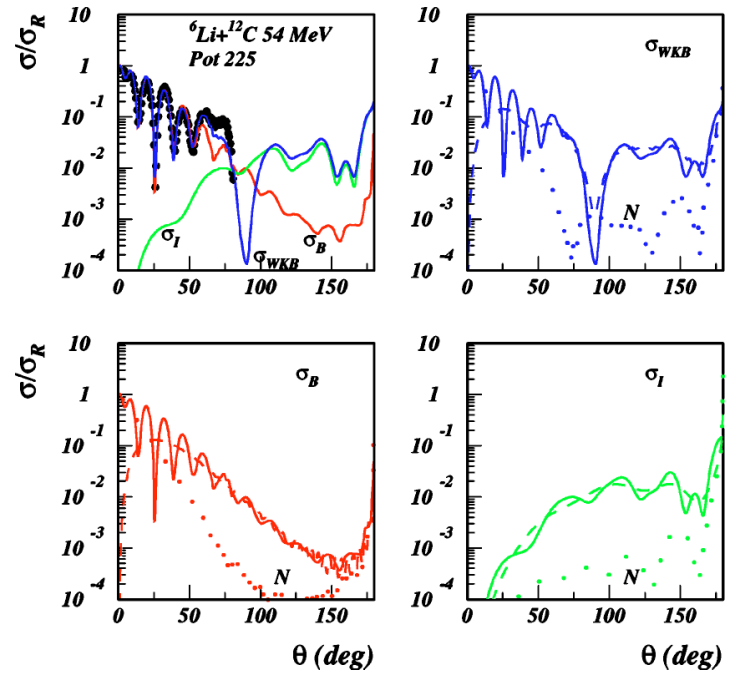

FIG. 13. (Color online) Semiclassical barrier and internal barrier decomposition of the cross section. The turning points and scattering function are those from Figs. 11 and 12, respectively. Each component is further dcomposed into far-side (dashed) and nearside (dotted) components. 


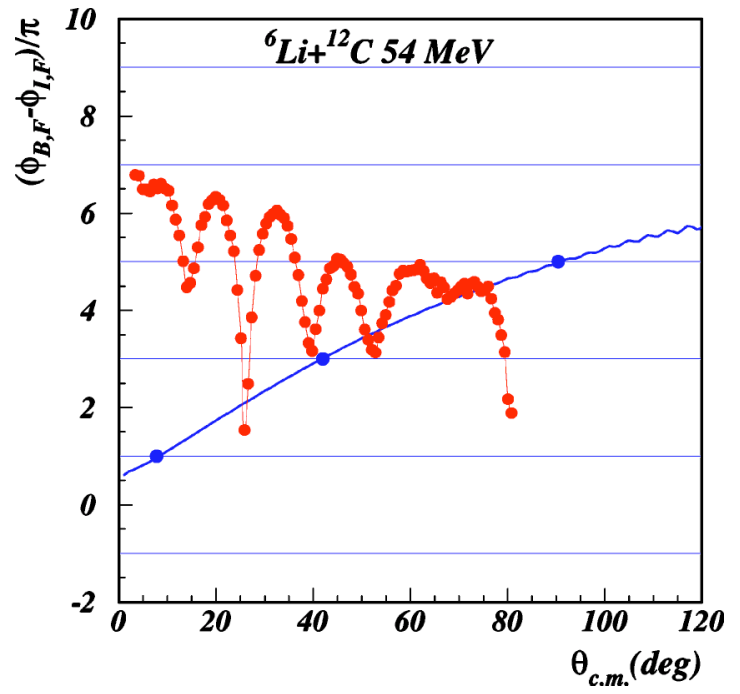

FIG. 14. (Color online) The phase difference of the far-side barrier and far-side internal barrier amplitudes as a function of scattering angle. Large dots indicate the predicted interference minima. For easier comparison, the experimental cross section is shown as $7+\log \left(\sigma / \sigma_{R}\right)$ to match the scale of the figure.

plitudes passes through an odd multiple of $\pi$-i.e., where minima should be expected. Since the crossing angle (where $\sigma_{B} \approx \sigma_{I}$ ) is about $\theta \approx 75^{\circ}$ and lies just in between predicted minima, the coherent interference around this angle gives rise to the "plateau" (constructive) and the deep minimum (destructive) at $\theta \approx 80^{\circ}$. Similar consideration apply to the other three reactions.

Thus, the intermediate angle exotic structure in angular distributions for the elastic scattering of ${ }^{6,7} \mathrm{Li}$ on light targets can be understood as a result of coherent interference of two far-side subamplitudes generated by different terms in the uniform multireflection expansion of the scattering function [terms $q=0$ and $q=1$ in Eq. (11)], corresponding to the scattering at the barrier and internal barrier. This interference effect appears as a signature of a surprisingly transparent interaction potential for loosely bound nuclei ${ }^{6,7} \mathrm{Li}$ which allows part of the incident flux to penetrate the nuclear interior and reemerge with significant probability.

\section{CONCLUSIONS}

We have performed measurements on extended angular ranges of the elastic scattering of loosely bound nuclei ${ }^{6,7} \mathrm{Li}$ on ${ }^{12,13} \mathrm{C}$ and ${ }^{9} \mathrm{Be}$ in four projectile-target combinations at $9 \mathrm{MeV} /$ nucleon and reanalyzed previous data for the scattering of ${ }^{7} \mathrm{Li}$ at $19 \mathrm{MeV} /$ nucleon in an effort to obtain systematic information on the interaction of $p$-shell nuclei with light targets. Optical potentials for these nuclei are needed for studies where highly peripheral transfer reactions involving radioactive nuclei are used as indirect methods for nuclear astrophysics and are an important factor in the accuracy and reliability of these methods. At the present time, the best information on the optical potentials for radioactive nuclei can be obtained only by extrapolation from adjacent less exotic nuclei. Our intention is to narrow the ambiguities in the optical model potentials by systematic studies of the scattering of loosely bound projectiles over a large range of angles and energies, and extract information that can be used for systems involving radioactive projectiles, for which elastic scattering data of very good quality are not easily available. We demonstrate this procedure by reanalyzing the oneneutron transfer reaction ${ }^{13} \mathrm{C}\left({ }^{7} \mathrm{Li},{ }^{8} \mathrm{Li}\right){ }^{12} \mathrm{C}$ using optical potentials obtained in the present study.

The present data, which extend over a much larger angular range than previously measured, confirm the existence of an exotic intermediate-angle structure, observed previously by Trcka et al. It was interpreted in Ref. [18] as a diffractive effect arising from an angular-momentum-dependent absorption. We adopt an opposite point of view and interpret these structures as refractive effects arising from a fine balance between the real and imaginary components of the optical potential. We have performed a traditional analysis of our data in terms of Woods-Saxon and microscopic JLM folded potentials. Both approaches lead to the conclusion that the optical potential is deep and surprisingly transparent, in line with findings for more bound systems. Folding model form factors have been renormalized in the usual way in order to account for the energy and radial dependence of the dynamic polarization potential. It is suggested that the DPP attains its maximum amplitude at approximately $16 \mathrm{MeV} /$ nucleon for these systems. The intermediate-angle structures could be reproduced only with potentials exceeding a critical volume integral of about $300 \mathrm{MeV} \mathrm{fm}^{3}$ and, consequently, are severely selective, limiting the ambiguities in the determination of the OMP. The remaining discrete ambiguities could be removed by a dispersion relation analysis. Based on a good estimation of the absorption at low energy (5-20 MeV/nucleon), this analysis allowed us to extract a smooth energy dependence of the optical potential. Our analysis did not find any spectacular anomaly near the Coulomb barrier and seems to confirm the conjecture of a canceling effect between the repulsive dynamic polarization potential due to the coupling with breakup channels and the attractive, dispersive component of the optical potential.

In our previous study [3] we found a simple recipe to obtain OMP for loosely bound $p$-shell nuclei from a doublefolding procedure using the JLM effective $N N$ interaction. The already independent real and imaginary parts were smeared with constant, but different ranges $t_{V}=1.2 \mathrm{fm}$ and $t_{W}=1.75 \mathrm{fm}$, which accounted for the well-known need for a wider imaginary potential to describe the experimental data. We found that a considerable renormalization of the real part was needed, $N_{V}=0.37 \pm 0.02$ (leading to volume integrals $J_{V} \simeq 220 \mathrm{MeV} \mathrm{fm}^{3}$ ), but not for the imaginary part $N_{W}$ $=1.00 \pm 0.09$. That recipe was already successfully applied to predict the elastic scattering angular distributions of RNBs on light targets in a number of cases at energies around $10 \mathrm{MeV} /$ nucleon. The present analysis shows that in order to reproduce the structures observed at intermediate angles in these cases, one needs to allow for a more complicated radial dependence of the dynamic polarization potential, which can be energy and target dependent, and requires deep real potentials with volume integrals larger than a critical value of $J_{V_{\text {crit }}} \approx 300 \mathrm{MeV} \mathrm{fm}{ }^{3}$. This is a conclusion of the phenomenological analyses and is supported by the dispersion rela- 
tion analysis. However, the elastic scattering data in the angular range of the Fraunhofer oscillations and the transfer reactions can be equally well described by the previous potentials produced by the folding procedure with fixed smearing ranges for the effective $N N$ interaction and the simple renormalization of Ref. [3], showing that the potentials are well described in the surface region. Extrapolation of the present optical potentials to heavier lithium isotopes proved to be successful as demonstrated by our study of a transfer reaction involving ${ }^{8} \mathrm{Li}$ and may work for ${ }^{9} \mathrm{Li}$ as well. The extremely fragile structure of the more exotic ${ }^{11} \mathrm{Li}$ may strengthen the interplay between the increased refractive power of the OM potential and the increased absorption at the surface. A simple extrapolation to this nucleus might not be useful. More accurate data and more complex models may be needed to clarify this point.

In an effort to clarify the reaction mechanism responsible for the intermediate-angle structures found at $9 \mathrm{MeV} /$ nucleon, we performed extensive semiclassical calculations within the uniform multireflection expansion of the scattering function of Brink and Takigawa. It has been shown that using complex trajectories, the (external-)barrier/ internal-barrier expansion is an exact realization of the dynamic decomposition of the quantum result into components responsible for that part of the incident flux reflected at the barrier and the part of the flux which penetrates into the nuclear interior and reemerges with significant probability. By combining the B/I decomposition with the usual far-side/ near-side expansion, we explain the intermediate-angle structure as a coherent interference effect of two subamplitudes (BF and IF). Thus, this refractive effect appears as a signature of a highly transparent interaction potential.

\section{ACKNOWLEDGMENTS}

This work was supported in part by the U.S. Department of Energy under Grant No. DE-FG03-93ER40773 and by the Robert A. Welch Foundation. One of the authors (F.C.) acknowledges the support of the Cyclotron Institute, Texas A \& $M$ University, and of the IN2P3 including that provided within the framework of the NIPNE-HH-IN2P3 convention.
[1] G. R. Satchler and W. G. Love, Phys. Rep. 55, 183 (1979).

[2] M. E. Brandan and G. R. Satchler, Phys. Rep. 285, 143 (1997).

[3] L. Trache, A. Azhari, H. L. Clark, C. A. Gagliardi, Y.-W. Lui, A. M. Mukhamedzhanov, R. E. Tribble, and F. Carstoiu, Phys. Rev. C 61, 024612 (2000).

[4] A. Azhari, V. Burjan, F. Carstoiu, C. A. Gagliardi, V. Kroha, A. M. Mukhamedzhanov, F. M. Nunes, X. Tang, L. Trache, and R. E. Tribble, Phys. Rev. C 63, 055803 (2001).

[5] X. Tang, A. Azhari, C. A. Gagliardi, A. M. Mukhamedzhanov, F. Pirlepesov, L. Trache, R. E. Tribble, V. Burjan, V. Kroha, and F. Carstoiu, Phys. Rev. C 67, 015804 (2003).

[6] Xiaodong Tang, A. Azhari, Changbo Fu, C. A. Gagliardi, A. M. Mukhamedzhanov, F. Pirlepesov, L. Trache, R. E. Tribble, V. Burjan, V. Kroha, and F. Carstoiu, Phys. Rev. C 69, 055807 (2004).

[7] J. Blackmon et al., Nucl. Phys. A (to be published).

[8] A. A. Ogloblin et al., Phys. Rev. C 62, 044601 (2000).

[9] S. Szilner et al., Phys. Rev. C 64, 064614 (2001).

[10] E. Stiliaris et al., Phys. Lett. B 223, 291 (1989).

[11] J. Knoll and R. Schaeffer, Ann. Phys. (N.Y.) 97, 307 (1976).

[12] R. Anni, Phys. Rev. C 63, 031601R (2001).

[13] H. Feshbach, Ann. Phys. (N.Y.) 5, 357 (1958); 19, 287 (1962).

[14] Y. Sakuragi, Phys. Rev. C 35, 2161 (1987).

[15] C. Mahaux, H. Ngo, and G. R. Satchler, Nucl. Phys. A449, 354 (1986).

[16] A. Nadasen et al., Phys. Rev. C 37, 132 (1988); and (private communication).

[17] A. Nadasen et al., Phys. Rev. C 47, 674 (1993); and (private communication).

[18] D. E. Trcka, A. D. Frawley, K. W. Kemper, D. Robson, J. D. Fox, and E. G. Myers, Phys. Rev. C 41, 2134 (1990).

[19] J. P. Jeukenne, A. Lejeune, and C. Mahaux, Phys. Rev. C 16,
80 (1977)

[20] D. M. Brink and N. Takigawa, Nucl. Phys. A279, 159 (1977).

[21] D. M. Pringle, W. N. Catford, J. S. Winfield, D. G. Lewis, N. A. Jelley, K. W. Allen, and J. H. Coupland, Nucl. Instrum. Methods Phys. Res. A 245, 230 (1986).

[22] D. H. Youngblood and J. B. Bronson, Nucl. Instrum. Methods Phys. Res. A 361, 37 (1995).

[23] D. H. Youngblood, Y.-W. Lui, H. L. Clark, P. Oliver, and G. Simler, Nucl. Instrum. Methods Phys. Res. A 361, 539 (1995).

[24] S. Kowalski and H. A. Enge, computer code RAYTRACE, 1986 (unpublished).

[25] L. Trache, A. Azhari, F. Carstoiu, H. L. Clark, C. A. Gagliardi, Y.-W. Lui, A. M. Mukhamedzhanov, X. Tang, N. Timofeyuk, and R. E. Tribble, Phys. Rev. C 67, 062801(R) (2003).

[26] A. M. Mukhamedzhanov et al., Phys. Rev. C 56, 1302 (1997).

[27] M. S. Hussein and K. W. McVoy, Nucl. Phys. A445, 123 (1985).

[28] A. Bonaccorso and F. Carstoiu, Nucl. Phys. A706, 322 (2002).

[29] M. E. Brandan and K. W. McVoy, Phys. Rev. C 55, 1362 (1997).

[30] M. Fukuda et al., Nucl. Phys. A656, 209 (1999).

[31] S. Kox et al., Phys. Rev. C 35, 1678 (1987).

[32] F. Carstoiu and R. J. Lombard, Phys. Rev. C 48, 830 (1993).

[33] F. Michel, G. Reidemeister, and S. Ohkubo, Phys. Rev. Lett. 89, 152701 (2002); Phys. Rev. C 63, 034620 (2001).

[34] M. Beiner and R. J. Lombard, Ann. Phys. (N.Y.) 86, 262 (1974).

[35] F. Carstoiu and R. J. Lombard, Ann. Phys. (N.Y.) 217, 279 (1992).

[36] E. Bauge, J. P. Delaroche, and M. Girod, Phys. Rev. C 58, 1118 (1998).

[37] X. Campi and D. W. L. Sprung, Nucl. Phys. A194, 401 (1972).

[38] Dao T. Khoa, G. R. Satchler, and W. von Oertzen, Phys. Rev. 
C 56, 954 (1997).

[39] M. F. Vineyard, J. Cook, K. W. Kemper, and M. N. Stephens, Phys. Rev. C 30, 916 (1984); (private communication).

[40] Yu. Gluchov et al., Report No. IAE-2989, 1978; and (private communication).

[41] D. P. Stanley, F. Petrovich, and P. Schwandt, Phys. Rev. C 22, 1357 (1980); and (private communication).

[42] K. Katori et al., Nucl. Phys. A480, 323 (1988).

[43] J. Cook, H. J. Gils, H. Rebel, Z. Majka, and H. KleweNebenius, Report No. KfK 3233, 1981.

[44] M. A. Nagarajan, C. C. Mahaux, and G. R. Satchler, Phys. Rev. Lett. 54, 1136 (1985).

[45] S. Kailas, Phys. Rev. C 41, 2943 (1990).

[46] M. A. Tiede, D. E. Trcka, and K. W. Kemper, Phys. Rev. C 44, 1698 (1991).

[47] N. Keeley, S. J. Bennett, N. M. Clarke, B. R. Fulton, G. Tun- gate, P. V. Drumm, M. A. Nagarajan, and J. S. Lilley, Nucl. Phys. A571, 326 (1994).

[48] C. Beck et al., Phys. Rev. C 67, 054602 (2003).

[49] F. Carstoiu and M. Lassaut, Nucl. Phys. A597, 269 (1996).

[50] A. Nadasen, T. Stevens, J. Farhat, J. Brusoe, P. Schwandt, J. S. Winfield, G. Yoo, N. Anantaraman, F. D. Becchetti, J. Brown, B. Hotz, J. W. Jänecke, D. Roberts, and R. E. Warner, Phys. Rev. C 47, 674 (1993).

[51] M. M. Gonzáles and M. E. Brandan, Nucl. Phys. A693, 603 (2001).

[52] M. F. Vineyard, J. Cook, and K. W. Kemper, Phys. Rev. C 31, 879 (1985).

[53] P. Fröbrich and R. Lipperheide, Theory of Nuclear Reactions (Clarendon Press, Oxford, 1996).

[54] K. W. Ford and J. A. Wheeler, Ann. Phys. (N.Y.) 7, 259 (1959). 